\title{
Key role of nuclear medicine in bringing imaging biomarkers into clinical practice
}

\author{
Ignasi Carrió
}

Published online: 5 November 2009

(C) Springer-Verlag 2009

Dear readers,

This year has brought compelling evidence of the central role of nuclear medicine in establishing imaging biomarkers in clinical decision making. Many articles published in imaging or clinical journals have reported how imaging biomarkers can be used to detect the predisposition for disease, screen for its presence, confirm its diagnosis, assess its severity, predict its response to available therapies and monitor its clinical course [1-4]. Currently, molecular imaging is witnessing a convergence and combination of many imaging modalities driven by unprecedented multidisciplinary collaboration among scientists. Nuclear medicine forms either the core or a crucial part of most of the resulting molecular imaging technologies. PET/MRI is an example of the new emerging multimodality molecular imaging techniques being used to interrogate the biology of various disease states [5].

As molecular imaging endpoints can be quantified, they are particularly useful for translational research but are also becoming critical for clinical care of patients. It is important to note that the biochemical biomarkers commontly used in clinical practice are typically quantitative. Therefore, the clinician may find it easier to integrate a quantitative imaging parameter as additional biomarker rather than a complicated set of images. FDG-PET, the first molecular imaging biomarker validated and applied to large patient populations, has already ben incorporated as surrogate endpoint in large clinical trials [6], but there are many areas where imaging biomarkers are being progressively introduced. Molecular mechanisms of increased metabolic activity of cancer cells offer opportunities for molecular

I. Carrió $(\bowtie)$

Barcelona, Spain

e-mail: icarrio@santpau.es imaging beyond FDG. Imaging of cell proliferation in monitoring response to therapy is being incorporated into new clinical trials. Molecular imaging of the apoptotic cascade and its regulation is important, as apoptosis might be desirable in some cancer treatment strategies but should be interrupted in other conditions such as heart failure. Various molecular imaging approaches are being proposed to assess metastatic potential and to differentiate indolent from aggressive phenotypes. Strategies to evaluate angiogenesis, hypoxia and tumor receptors will play a role in new forms of personalized cancer treatments. Molecular imaging is bringing more specific tracers targeting cellular and subcellular biologic events, which are expected to shed more light upon the mechanisms by which stem cells confer benefit in various diseases.

Overall, these developments provide a constellation of opportunities for molecular imaging techniques to offer powerful biomarkers in research and clinical care. Although individual molecular imaging techniques often compete to become the only means to address a given biologic problem, in most circumstances appropriate combinations of the wide spectrum of molecular imaging modalities provide a powerful integration that transcends the single perspective given by any one technique. The resulting integrated imaging technologies should bring more effective biomarkers and should bridge the newly discovered biologic phenomena with new integrated, targeted and tailored therapies. Again, nuclear medicine has the unique opportunity to understand and lead such evolutionary processes.

As a global vehicle for scientific communication in the field, the European Journal of Nuclear Medicine and Molecular Imaging (EJNMMI) has continued its progress in 2009. Published items over the past year include: 34 Editorials, 221 Original Articles, 6 Review Articles, 16 
Images of the Month, 31 Letters, 6 Guidelines and 11 Focus On... articles. An innovative supplement on PET/MR, edited by Dr. Thomas Beyer and Dr. Bernd Pichler, was published early in the year [5]. Focussed editorial commentaries written by expert leaders help the readers to put special papers into appropriate perspective. Original Articles that portray the best clinical and basic research performed in our field are selected from more than 1,000 submissions coming from all over the world. The Review Articles, edited by Prof. Angelika Bischof Delaloye, deliver comprehensive reviews of relevant topics. Prof. Luc Mortelmans selects from among the hundreds of submitted Images of the Month those with the highest priority in terms of reader interest and clinical relevance. The Guidelines reflect the work of the EANM committees and expert bodies and are central in defining appropriate procedures and best practice. The Focus On... section, as developed by Prof. Giovanni Lucignani, offers updates on interesting and hot topics from the recent medical literature. The Editorial Office, Associate Editors and Editorial Board are always committed to provide EJNMMI's readers with the best in the field of nuclear medicine and molecular imaging.

The acceptance rate for EJNMMI is now below 20\%. This figure comes after a complex peer review process that includes selection of appropriate expert referees, thoughtful and detailed review, balanced evaluation of relevance and priority, and the final editorial decision. All of us who are involved in this process fully realize the effort that goes into a submission and are aware, from our personal experience as authors, of the disappointment when a manuscript is rejected after review. Appropriate peer review, a most difficult and serious task, is crucial to make the difference between a good or poor scientific journal. Here, once again, I would like to express my recognition to the expert referees who have participated in the EJNMMI review process, in particular those who do not belong to the Editorial Board. Over 1,200 experts from all over the world have participated in the 2009 EJNMMI review process. In the name of the Editorial Board, the authors and the readers, my sincere gratitude goes to them all.

A few figures will help to illustrate this year's journal performance. The average time from submission to first decision in 2008 was 26 days; the average time from submission to final acceptance was 65 days (including revision and re-submission); and that from submission to rejection was 24 days. Rapid selection of appropriate reviewers and delivery of the reviews within the allotted time of 2 weeks is crucial. The EJNMMI expert database contains more than 1,000 names, coming from a search across the literature based upon relevant keywords. Authors are therefore recommended to indicate the keywords that most accurately reflect their work in order to prompt selection of the most suitable reviewers. Once a manuscript is accepted, it rapidly becomes available online and can be consulted and cited. EJNMMI indices in 2007 continue to show that the journal is advancing. The 2008 impact factor rose to 4.532 (1,822 citations of 402 articles), the highest ever. Our immediacy index (citations to articles published in the same year) has gone up to 0.962 , which reflects improved visibility of and easier online access to the EJNMMI articles and helps citation of our articles within the time window of two years required for the impact factor calculation [7]. The cited half-life (number of publication years from the current year which account for $50 \%$ of current citations) is 5.7 years and the total number of citations in 2008 increased to 8,825 (Journal Citation Reports, Thompson Institute for Scientific Information).

To acknowledge excellence in clinical and basic research in the field, the EJNMMI has presented its annual awards. An article on regional analysis of FDG and PIB-PET images in normal aging, mild cognitive impairment, and Alzheimer's disease from researchers in New York, Jinan and Turku [8] was selected as the best clinical paper in 2008, underlining the role of PET in the identification of potential survivors. This paper, by Yi Li et al., using an automated image analysis system, provides extensive description of the use of FDG and PIB in mild cognitive impairment and Alzheimer's disease and shows that the combination of the two modalities improves the diagnostic accuracy for mild cognitive impairment. The award for best basic science paper in 2008 went to a group in Bethesda for an article describing a new affibody molecule as new molecular tracer for in-vivo monitoring of HER2 expression by PET from [9]. This paper, by Gabriela Kramer-Marek et al., describes a new affibody radioconjugate that can be used to assess HER2 expression in vivo by PET imaging and might be used to monitor changes of receptor expression in response to therapeutic interventions. On the other hand, the most cited EJNMMI paper in 2008 (from articles published in 2005 and most cited between 2005 and 2008) was an article from Zurich on the value of PET, CT, and in-line PET/CT in patients with gastrointestinal stromal tumours (GIST) and its role in assessment of long-term outcome in patients treated with imatinib mesylate [10]. This paper, by G.W. Goerres et al., provides evidence on the prognostic value and impact on clinical decision making of PET/CT as a major imaging biomarker in GIST patients. I would like to note that most cited EJNMMI papers in ISI WOS reflect diverse scientific activity and show that the EJNMMI impact factor is not based upon a few very highly cited articles but on a solid majority of articles with intermediate/high rates of citation.

At the end of the year, my personal thanks again have to go to those people who are key in the functioning of the 
journal: Carmina Jimenez, the new editorial assistant, who takes care of an appropriate flow of manuscripts across the system; Anneliese Sand, who is responsible for a smooth production process; Claudia Schiffers, who takes care of the journal's website; and Sabine Ben Ghechir who coordinates editorial activities at Springer. Dear colleagues and readers, I hope that the role of nuclear medicine and molecular imaging in research and clinical practice will continue expanding next year, and wish you all a very successful 2010 !

\section{Ignasi Carrió}

EJNMMI Editor-in-Chief

\section{References}

1. Larson SM. Practice-based evidence of the beneficial impact of positron emission tomography in clinical oncology. J Clin Oncol. 2008;26(13):2083-4.

2. Hachamovitch R. Assessing the prognostic value of cardiovascular imaging: a statistical exercise or a guide to clinical value and application. Circulation. 2009;120(14):1342-4.
3. Visser PJ, Knopman DS. Amyloid imaging in the prediction of Alzheimer-type dementia in subjects with amnesic MCI. Neurology. 2009;73(10):744-5.

4. Hosking CR, Schwartz JL. The future's bright: Imaging cell biology in the 21st century. Trends Cell Biol 2009; ahead of print.

5. Beyer T, Pichler B. Eur J Nucl Med Mol Imaging. 2009;36:S1144.

6. Lordick F, Ott K, Krause B. PET to assess early metabolic response and to guide treatment of adenocarcinoma of the oesophagogastric junction: the MUNICON phase II trial. Lancet. 2007;8(9):797-805.

7. Carrió I. Of Impact, metrics and ethics. Eur J Nucl Med Mol Imaging. 2008;35:1049-50.

8. Li Y, Rinne JO, Mosconi L, et al. Regional analysis of FDG and PIB-PET images in normal aging, mild cognitive impairment, and Alzheimer's disease. Eur J Nucl Med Mol Imaging. 2008;35:2169-81.

9. Kramer-Marek G, Kiessewetter DO, Martinova L, et al. (18F) FBEM-ZHER2:342-Affibody Molecule- a new molecular tracer for in-vivo monitoring of HER2 expression by positron emission tomography. Eur $\mathrm{J}$ Nucl Med Mol Imaging. 2008;35:1008-18.

10. Goerres GW, Stupp R, Barghouth G, et al. The value of PET, $\mathrm{CT}$, and in-line PET/CT in patients with gastrointestinal stromal tumours: long-term outcome of treatment with imatinib mesylate. Eur J Nucl Med Mol Imaging. 2005; $32: 153-62$. 